\title{
Imaging findings in craniofacial childhood rhabdomyosarcoma
}

\author{
Nicole J. M. Freling • Johannes H. M. Merks • \\ Peerooz Saeed • Alfons J. M. Balm • Johannes Bras • \\ Bradley R. Pieters • Judit A. Adam • Rick R. van Rijn
}

Received: 11 February 2010 /Revised: 7 June 2010 /Accepted: 14 June 2010 /Published online: 20 August 2010

(C) The Author(s) 2010. This article is published with open access at Springerlink.com

\begin{abstract}
Rhabdomyosarcoma (RMS) is the commonest paediatric soft-tissue sarcoma constituting 3-5\% of all malignancies in childhood. RMS has a predilection for the head and neck area and tumours in this location account for $40 \%$ of all childhood RMS cases. In this review we address the clinical and imaging presentations of craniofacial RMS, discuss the most appropriate imaging techniques, present characteristic imaging features and offer an overview of differential diagnostic considerations. Post-treatment changes will be briefly addressed.
\end{abstract}

\author{
N. J. M. Freling $(\bowtie) \cdot R$. R. van Rijn \\ Radiology Department, Academic Medical Centre, \\ Meibergdreef 9 , \\ Amsterdam 1105 AZ, The Netherlands \\ e-mail: n.j.freling@amc.uva.nl

\section{J. H. M. Merks} \\ Paediatric Oncology, Academic Medical Centre, \\ Amsterdam, The Netherlands \\ P. Saeed \\ Orbital Surgery and Ophthalmology, Academic Medical Centre, \\ Amsterdam, The Netherlands
}

\section{A. J. M. Balm}

Head and Neck Oncology, Netherlands Cancer Institute,

Amsterdam, The Netherlands

J. Bras

Pathology, Academic Medical Centre,

Amsterdam, The Netherlands

\section{B. R. Pieters}

Radiotherapy, Academic Medical Centre,

Amsterdam, The Netherlands

\section{J. A. Adam}

Nuclear Medicine, Academic Medical Centre,

Amsterdam, The Netherlands
Keywords Rhabdomyosarcoma $\cdot$ Orbit $\cdot$ Face $\cdot$ US · CT . MRI · Child · Paediatric head and neck · MR imaging · Review

\section{Introduction}

Rhabdomyosarcoma (RMS) is the commonest paediatric soft-tissue sarcoma, constituting 3-5\% of all malignancies in childhood [1]. RMS has a predilection for the head and neck area and tumours in this location account for $40 \%$ of all childhood RMS cases, making it the second most common primary malignant head and neck tumour in children after malignant lymphoma [2]. RMS is a highgrade, malignant mesenchymal tumour probably originating from primitive mesenchymal cells that can arise anywhere in the body, including sites where striated muscle is not normally found.

RMS is predominantly a disease of childhood and occurs somewhat less frequently in adolescents. The mean age at diagnosis is $5-6$ years and $72-81 \%$ of patients are younger than 10 years $[3,4]$. In $5-6.5 \%$ the child is younger than 1 year and only $2 \%$ of RMS manifests at birth $[5,6]$. There is a slight male predominance, $\mathrm{M}: \mathrm{F}=1.3: 1$.

Clinical symptoms vary and depend on the location of the primary tumour and the presence or absence of metastases [7, 8]. Head and neck RMS (HNRMS) can present indolently, with nonspecific or minimal signs and symptoms, leading to a delay in diagnosis. For example, chronic otitis or nasal obstruction may be the presenting symptom in a nasopharyngeal location whereas persistent headache may be a sign of intracranial tumour extension. More ominous signs are cranial nerve palsies in parameningeal locations indicating involvement of the skull base. Orbital tumours are detected relatively early, due to 
their obvious symptoms and this possibly relates to better prognosis of orbital RMS. Although proptosis is the result of an intra-orbital tumour location, it can also be caused by orbital invasion from another compartment. A prolonged duration of common symptoms or a rapidly growing mass should arouse suspicion of a malignant lesion.

Three main groups of RMS are recognized, based on histological morphology: embryonal, alveolar and pleomorphic, of which the former two mainly occur in children and the last only in adults [8]. The embryonal type (eRMS) accounts for about $70 \%$ of cases, affects somewhat younger patients and has a better prognosis. Alveolar RMS accounts for about $15 \%$, is seen in older children and has a worse prognosis $[9,10]$. Three different primary locations of HNRMS are recognized: parameningeal (PM), nonparameningeal head and neck (NPM) and orbital (ORB), which are involved in approximately $50 \%, 25 \%$ and $25 \%$ of cases respectively (Table 1) [9]. Parameningeal location carries a worse prognosis [5-year overall survival (OS) 6473\%] compared to NPM and ORB locations (5-year OS $64-80 \%$ and $85-88 \%$ respectively) for at least two reasons $[4,9,11]$. Firstly, the deep location may conceal a tumour that remains clinically silent unless it has grown to a considerable size and becomes visible from the outside, and secondly because the PM location is very close to the skull base and contains vital structures such as the internal carotid artery, internal jugular vein and multiple cranial nerves, precluding oncological resection. A third reason might be that PM RMS has a different biological composition. In a series of 526 children with PM RMS, Raney et al. [4] found cranial nerve palsy and/or cranial base bony erosion in $33 \%$ of children at diagnosis, while $35 \%$ presented with intracranial extension in contiguity with the primary tumour. Only $32 \%$ had no signs of meningeal involvement.

Imaging provides crucial information early in the diagnosis by defining the exact location of the lesion and offering a differential diagnosis, thereby guiding the subsequent steps of the diagnostic analysis and follow-up. Furthermore it makes an essential contribution in the staging of HNRMS, showing the size and location of the tumour and its extension into adjacent compartments, as it can demonstrate the presence or absence of intracranial extension, involvement of cranial nerves, cranial base bony erosion, orbital involvement and encasement of the carotid or vertebral arteries [12, 13].

The detection of lymph node metastases has also become more accurate with modern imaging techniques, as has the demonstration of distant metastases [14-19].

Sometimes imaging helps to guide a biopsy procedure by showing the best route to obtain representative material.

Follow-up imaging is important to monitor tumour regression during or after completion of chemotherapy and to detect tumour recurrence [19-21].
In this review we address the clinical and imaging presentations of craniofacial RMS, discuss the most appropriate imaging techniques, present characteristic imaging features and offer an overview of differential diagnostic considerations. Post-treatment changes will be briefly addressed.

\section{Imaging techniques}

\section{MRI}

Unlike small superficial mass lesions, which are welldemonstrated by US, tumours of the head and neck in the paediatric population are currently best depicted with MRI. Superior soft-tissue contrast, excellent spatial resolution, multiplanar imaging and the absence of potentially harmful ionizing radiation make MRI a powerful instrument for accurate local staging. With dedicated coils, MRI can cover the whole area comprising the skull base and intracranial compartment as well as the face, the orbits and the neck, thereby assessing local extension of the primary tumour and involvement of regional nodes. Whole-body MRI (WBMRI) may replace bone scintigraphy in the coming years to assess distant metastases [14, 15].

Although the advantage of MRI in this young patient population is its lack of ionizing radiation exposure, a major drawback is the long examination time (up to 30 $45 \mathrm{~min}$ ) and the fact that MRI is extremely sensitive to motion artefacts. Therefore most children younger than $6-$ 7 years need sedation or general anaesthesia during their MRI examination.

MRI protocols are reproducible and therefore MRI is suitable to assess response during chemotherapy, to depict residual mass lesions after completion of therapy and to detect and assess loco-regional and distant recurrent disease during follow-up.

\section{MRI techniques}

Routine spin-echo (SE) T1- and T2-weighted sequences before and after intravenous (IV) contrast administration in axial, coronal and/or sagittal planes are crucial for evaluation of a head and neck tumour in children.

Diffusion-weighted imaging (DWI) seems to be a promising new tool to differentiate benign from malignant lesions in the paediatric population [12, 22]. However, most of the literature concerns adult patients with head and neck malignancies and there still is some controversy about its accuracy [23-25]. DWI is reported to be superior to conventional methods in recognizing malignant neck nodes in adult patients with squamous cell carcinoma of the head and neck $[18,26,27]$. It has also been reported to be a 
Table 1 Locations of HNRMS

\begin{tabular}{lll}
\hline PM & Non-PM & Orbital \\
$25-50 \%$ & $25 \%$ & $25 \%$ \\
\hline $\begin{array}{l}\text { Nasopharynx } \\
\text { PPS/MS/pterygopalatine } \\
\text { fossa }\end{array}$ & Scalp & $\begin{array}{c}\text { Eye lids } \\
\text { Lacrimal } \\
\text { gland }\end{array}$ \\
$\begin{array}{l}\text { Nasal cavity and paranasal } \\
\text { sinuses }\end{array}$ & Oral cavity & $\begin{array}{c}\text { Extra-ocular } \\
\text { muscles }\end{array}$ \\
$\begin{array}{l}\text { Middle ear } \\
\text { Orbital tumours with bone } \\
\text { erosion }\end{array}$ & Larynx & Globe \\
$\begin{array}{l}\text { Involvement of vessels } \\
\text { (carotid artery, jugular vein) } \\
\text { and cranial nerves } \\
\text { with direct intracranial } \\
\text { connection }\end{array}$ & Oropharynx & Orbit \\
$\begin{array}{l}\text { All tumours with cranial } \\
\text { nerve paresis }\end{array}$ & Cheek & \\
$\begin{array}{l}\text { All tumours with intracranial } \\
\text { and/or intraspinal extension }\end{array}$ & Thyroid/ & \\
$\begin{array}{l}\text { CSF tumour-cell-positive } \\
\text { patients }\end{array}$ & parathyroid & \\
\hline
\end{tabular}

${ }^{a}$ After EpSSG $2005[3,9,76]$

valuable tool in the analysis of post-treatment residual disease, where DWI may differentiate postoperative scarring or necrosis from residual viable or recurrent tumour [28]. At this moment no evidence-based data are available specifically concerning DWI in HNRMS.

Gradient-echo (GRE) sequences, often used in brain imaging, are short three-dimensional sequences with thin $(<1 \mathrm{~mm})$ slices but are not very useful in the head and neck area due to artefacts from air-bone interfaces. Signal contrast between tumour and normal soft tissues is reduced, especially in the orbit, resulting in poor lesion conspicuity. Major advantages include their multiplanar reconstruction possibility.

Volumetric interpolated breath-hold examination (VIBE - Siemens, Erlangen, Germany) is a T1-W, 3-D Flash, fatsaturated sequence with a very homogeneous fat saturation effect. It is used after IV contrast, is very fast to acquire (30 s) and 3-D reconstructions are possible from the original acquisition plane. Therefore, VIBE may be of help to detect subtle skull base abnormalities and perineural tumour spread [29].

$1 \mathrm{H}$-spectroscopy is an MRI technique that has been used to identify brain tumours and to differentiate malignant from benign disease. Tissue volumes of at least $1 \mathrm{~cm}^{3}$ are sampled and analyzed for spectral elements. However, the presence of many different tissues in even a small volume sample in the head and neck area still limits the use of MR spectroscopy on a routine basis.

Dynamic contrast-enhanced MR (DCE-MRI) imaging is a technique to assess tumour vascularity in vivo. It has been applied to differentiate benign from malignant disease, where some overlap exists between highly vascular benign tumours and malignancies and has been shown to be useful to correctly predict response to therapy in histologically proven soft-tissue and bone sarcomas [12, 30]. Early, rapidly progressive enhancement was correlated histologically with residual viable tumour. Late, gradual enhancement or the absence of enhancement was correlated with necrosis or granulation tissue [31]. DCE-MRI may be of interest in assessing the nature of a residual mass after completion of treatment [32].

\section{Multidetector CT (MDCT)}

With modern spiral CT scanners examination time is reduced from minutes to seconds. Multiplanar reconstructions and the automatic tailoring of machine settings according to the volume of the patient have led to a substantial reduction of radiation dose compared to the former single-slice scanners but it is still not zero [33].

In patients with RMS, CT is mainly performed to assess the absence or presence of lung metastases [8].

When using CT we must be aware that exposure to ionizing radiation may theoretically induce a second potentially fatal cancer in children who have already manifested a tendency to harbour cancer [34, 35].

\section{US}

US is probably the most commonly applied cross-sectional imaging technique in children. Unfortunately, US cannot reach the deep compartments of the face, hampered as it is by bone and air, and therefore US has severe limitations for the evaluation of primary head and neck tumours. However, US may be invaluable to obtain an initial quick confirmation of a suspected mass lesion and of its cystic, solid or vascular nature.

Table 2 MRI protocol for HNRMS at 1.5-T

Head-neck coil

Axial SE T1-W, SE T2-W

Coronal SE T1-W; optional: sagittal SE T1-W

After IV gadolinium: coronal without fat saturation

axial, coronal, sagittal with fat saturation

Slice thickness: $\leq 3 \mathrm{~mm}$

Field of view 180-200

DWI-echo planar imaging or non-EPI

Optional:

T1- and/or T2-W fat-saturated sequences (STIR, SPIR)

Contrast-enhanced GRE sequences (VIBE)

General anaesthesia in young children: $<7$ years

For orbital RMS it is recommended to add fat saturation in all contrast-enhanced series and to incline the sagittal series parallel to the optic nerve 
Fig. 1 Masticator space eRMS in a 4-year-old boy presenting with a rapidly progressive right side preauricular mass. a Coronal SE T1-W image without IV contrast demonstrates obliteration of the fat planes (arrow) of the MS and the PPS and of the sphenoid wing on the right side [compare to the normal left side (white arrow)]. Also note abnormal configuration and signal of the right mandible, better shown on the axial SE T1-W images (b). b Axial SE T1-W image shows tumour bulging through the mandible underneath the masseter muscle with preservation of the fat plane (arrowhead). The PPS is compressed and the fat is displaced posteriorly and medially (arrow), indicating that the origin of the tumour is not in the PPS but in the MS. Extension of tumour through the stylomandibular foramen into the parotid gland (small arrow). c The mass is of high signal intensity throughout on axial SE T2-W sequence. d Coronal SET1 image after i.v. contrast: heterogeneous enhancement with many necrotic areas. Tumour extention through the foramen ovale into the right cavernous sinus (large arrow). Note also subtle dural enhancement (small arrow), which was interpreted as tumour extension, but might have been reactive changes only, according to thickness $<5 \mathrm{~mm}$
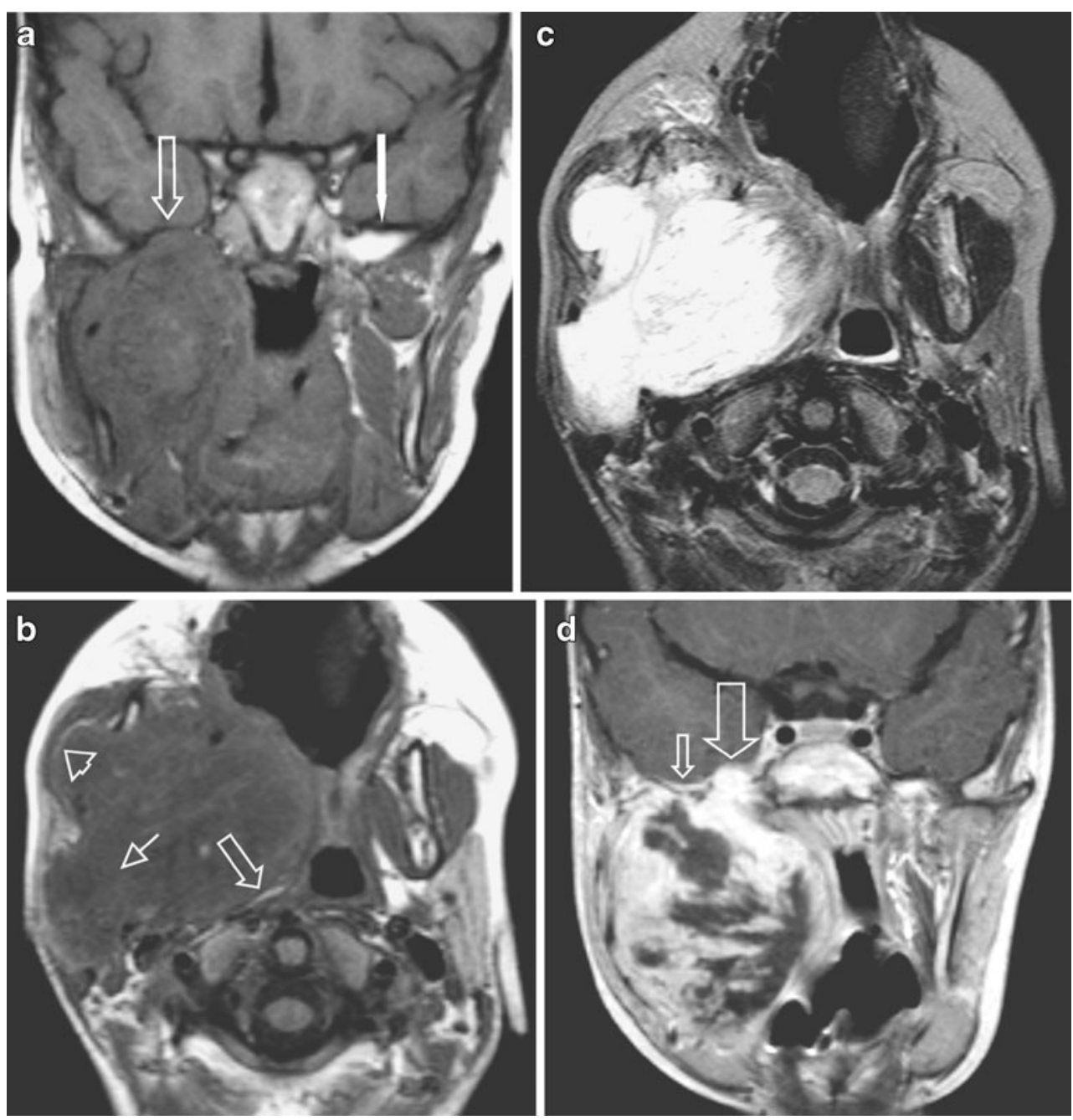

The superficial neck, however, is well-depicted by US. US is very accurate in delineating neck masses and enlarged or otherwise suspicious lymph nodes in children with HNRMS and is useful to guide fine-needle aspiration cytology (FNAC). For primary tumours open biopsies under general anaesthesia are preferred and in the same session FNAC of suspicious nodes can be performed. Often children are referred from another centre, where a biopsy of the primary tumour has already been performed. Those children will have their staging under general anaesthesia, including a bone marrow puncture and trephine, and a lumbar puncture for PM cases; the same anaesthesia can then be used to perform FNAC of suspicious nodes, if present.

\section{Bone scintigraphy}

Although bone scintigraphy is currently required as part of the work-up of children with RMS [European paediatric soft-tissue sarcoma Study Group (EpSSG) and Children's Oncology Group (COG) protocols], there is doubt about its yield in routine use, which is said to be very low [14, 36]. Bone scintigraphy is most accurate for osteoblastic metastases, whereas PET-CT is most effective in demonstrating osteolytic metastases. Therefore in future protocols bone scintigraphy might be considered for symptomatic or highrisk patients only.

\section{PET-CT}

${ }^{18}$ FDG PET-CT allows examination of the entire body in one session and can detect not only the primary tumour but also regional and distant metastatic disease [14]. Tateishi et al. [37] reported on 35 patients in a blinded retrospective study, in whom PET-CT had the same accuracy in staging the primary tumour (T-stage) and regional lymph nodes (N-stage) as conventional imaging (radiographs, whole-body CT and/or MRI) [37]. However, distant metastases (M-stage) were much better appreciated by PET-CT compared to MRI, CT or bone scintigraphy. In a non-blinded retrospective study Klem et al. [38] found that PET did detect occult lymph node metastases in the neck not identified by other imaging techniques. 


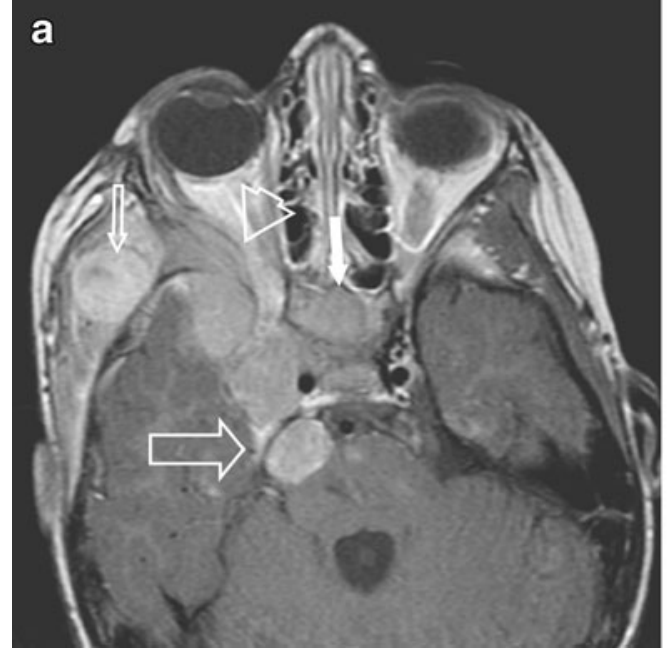

Fig. 2 Four-year-old girl, presenting with a right side preauricular mass and cranial nerve palsy (ptosis). A huge eRMS originating within the MS was found with involvement of the greater wing of the sphenoid bone and orbital apex on the right side, with massive perineural extention along the 2nd and 3rd branches of the right trigeminal nerve and involvement of the cisternal portion. a Axial contrast-enhanced SE T1-W image shows a mass within the masticator space (small arrow), abnormal enhancement of the sphenoid wing (arrowhead), the clivus (white arrow) and multiple masses along the

However, the detection of bone metastases by PET was, surprisingly, equal to conventional techniques (MRI, CT, radiograph). For the evaluation of lymph node metastases, Völker et al. [17] found a higher sensitivity of FDG-PET compared to conventional imaging, $88 \%$ and $62 \%$ respectively. This group also showed that FDG-PET correctly identified all bone metastases in 10 patients, whereas conventional imaging was falsely negative in 3 patients.

Whether WBMRI or PET-CT should be used in routine staging procedures in the paediatric population is still unclear. Only a prospective clinical trial implementing both imaging techniques combined with bone scintigraphy will be able to answer this question. In our clinic we do not perform PET-CT on a routine basis in children with HNRMS, due to the radiation exposure and less accurate performance in assessing the primary tumour. In our clinic, only children with an increased risk of distant metastases are considered as candidates for low-dose PET-CT or WBMRI. Most children with metastatic disease are old enough to co-operate well; younger or uncooperative children are sedated using chloral hydrate and closely monitored.

\section{Staging HNRMS}

With continued improved survival of children with RMS, correct assessment of the disease at presentation could lead to significant clinical benefit.

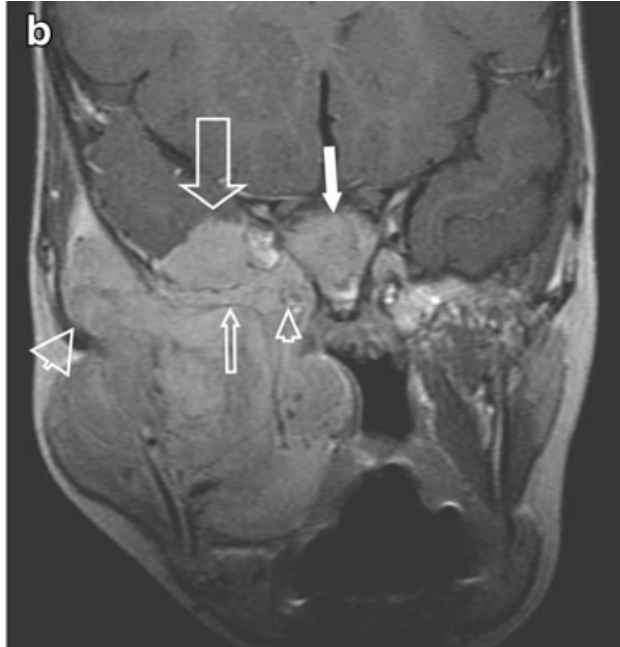

2nd branch of the right trigeminal nerve (pterygopalatine fossa, foramen rotundum, cavernous sinus) extending into the posterior fossa with involvement of the cisternal portion of the trigeminal nerve (large arrow). b Coronal contrast-enhanced SE T1-W image of the same patient, shows involvement of the right MS, along the temporalis muscle (large arrowhead), into the masseter muscle. Tumour extends into the foramen rotundum (small arrowhead), the sphenoid wing (small arrow) with massive dural invasion $(>5 \mathrm{~mm}$ ) (large arrow) and invasion of the clivus (white arrow)

Accurate staging, therefore, has become a major goal before choosing the optimal treatment strategy in children with HNRMS [8, 38]. Uniform staging is important in comparing results from different co-operative groups as it may lead to different treatment stratifications and protocols in future trials, aiming to continuously improve treatment results. The main staging system is the postsurgical system developed by the Intergroup Rhabdomyosarcoma Study Group (IRSG), which is currently used by both EpSSG and COG [8].

\section{Primary location $(\mathrm{T})$}

A routine MR imaging protocol (Table 2) includes SE T1-W and SE T2-weighted axial, coronal and sagittal images before and after intravenous (IV) contrast administration. Axial and coronal planes are used for optimal assessment of local extension and invasion of adjacent compartments, whereas coronal and sagittal contrastenhanced images address questions about involvement of the skull base and intracranial (dura, brain) and/or perineural extension (Figs. 1 and 2). Additional DWI series are recommended in all patients.

Imaging must assess the origin and local extension of the primary tumour, its size (in three dimensions, in $\mathrm{cm}$ ), the presence or absence of tumour extension beyond the skull base, invasion of the anterior, middle and posterior cranial fossa, abnormal dural enhancement, perineural enhance- 
Table 3 Checklist for the radiology report

PM: consider additional CT in selected patients
- Origin: PM; head and neck; orbit

- Extension adjacent compartments:

PPS

MS

Pterygopalatine fossa

Orbit

Paranasal sinuses

- Extension foramen ovale, foramen rotundum, other

- Cavernous sinus involvement

- Perineural enhancement: N-V, N-VII, other

- Intracranial extension:

Anterior cranial fossa

Middle cranial fossa

Posterior cranial fossa

- Dural enhancement: $<$ or $>5 \mathrm{~mm}$ thickness

- External auditory canal/middle ear

- Mastoid/internal auditory canal

- Maximum sizes in 3 dimensions in $\mathrm{cm}$

- Suspicious lymph nodes

- Retropharyngeal space

- Neck

Bilateral/unilateral

Homolateral/contralateral

- Bone changes (skull base, mastoid, petrous bone, mandible, maxilla, orbit, zygoma, skull vault) ment along cranial nerves and intracerebral enhancement (Table 3).

\section{Perineural spread}

Perineural spread is said to occur when abnormal enhancement along cranial nerves $(\mathrm{CN})$ is observed, either in isolation or in combination with nodular thickening, in patients with malignant head and neck tumours [39-43]. Most often involvement of the trigeminal nerve branches and/or the facial nerve is seen, but any other cranial nerve may be involved.

Perineural involvement of the trigeminal nerve may be identified by abnormal enhancement along its course from the brainstem into the cavernous sinus. From this point abnormal enhancement along the three trigeminal branches (V-1, V-2, V-3) should be looked for carefully: first the ophthalmic nerve (V-1) in the upper quadrant of the orbit, then the maxillary nerve (V-2) within the foramen rotundum, the infraorbital fissure, along the lateral floor of the orbit (infraorbital sulcus) through the infraorbital foramen and finally the mandibular nerve (V-3) within the foramen ovale, the masticator space (MS) and the mandibular canal up to the mental foramen. It is helpful to compare with the uninvolved side to detect subtle asymmetry. Perineural enhancement also may be seen along the facial nerve as far as the inner auditory canal. The pterygopalatine fossa, being an anatomical crossroad, also should be scrutinized for obliteration of normal fat planes and abnormal enhancement of the pterygopalatine ganglion, the palatine nerves as well as the vidian nerve, which extends from the pterygo-

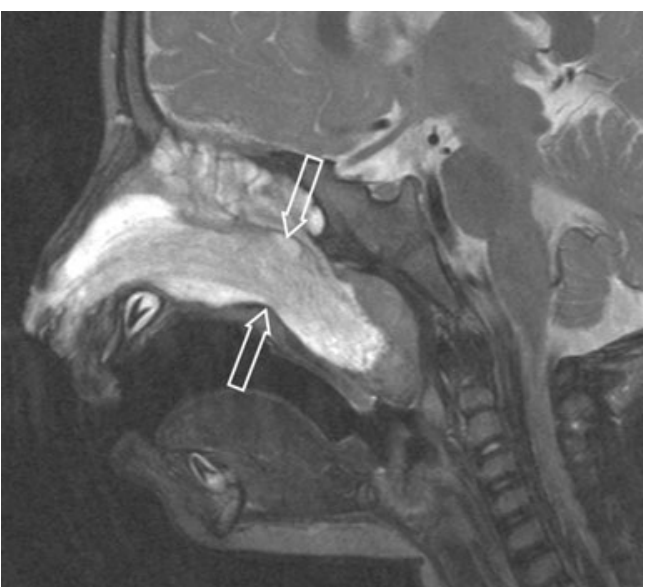

Fig. 3 One-year-old boy presenting with a rapidly growing mass from the right nasal ostium. Sagittal T2-W MRI shows a well-defined, sausage-like lesion with heterogeneous signal intensity (arrows). The high signal intensity of the ethmoid cells was interpreted as obstructed secretions rather than direct tumour invasion in the absence of obvious destruction of the ethmoid air cells. At surgery, after three courses of chemotherapy, no tumour was found in the ethmoids; a small residual tumour was adherent to the skull base, not identified on preoperative MRI, classifying the tumour as a PM location 


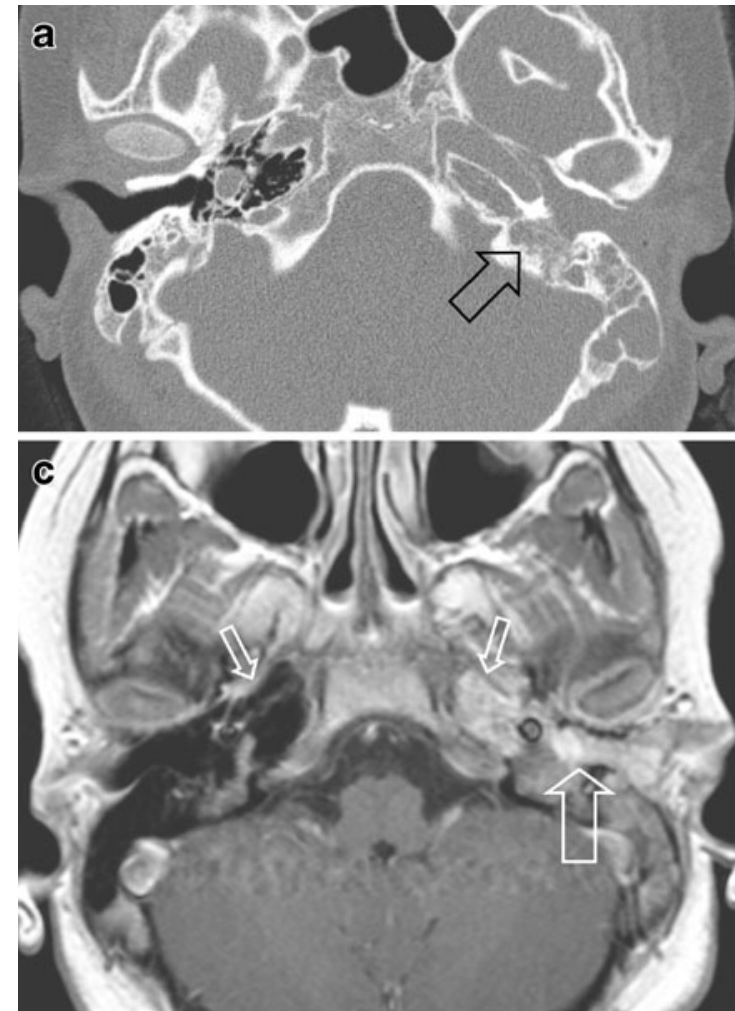

Fig. 4 Left external auditory canal (EAC) location in a 10 -year-old girl with itching and pain of the left ear for 2 weeks and conductive hearing loss. No history of acute or chronic middle ear disease. At clinical examination a soft-tissue mass was seen, completely filling the EAC. a CT demonstrates unilateral complete opacification of the EAC and the middle ear cavity on the left side with erosion of the bony walls medially (arrow). The carotid canal wall is intact as is the posterior aspect of the mastoid. Note the asymmetrical pneumatisation of the petrosal apex, a normal variant. b Coronal T1-W MRI shows an

palatine ganglion through the vidian canal posteriorly and communicates with the greater petrosal nerve.

In one of our patients we noticed abnormal enhancement of the thickened $\mathrm{CN} \mathrm{V-2}$ within the foramen rotundum at diagnosis. During chemotherapy the swelling regressed but enhancement lasted for many years during follow-up without clinical symptoms, probably due to mild inflammatory changes (chronic neuritis). It seems therefore that enhancement per se is not always due to tumour extension and cautious interpretation of persistent abnormal enhancement, without concomitant increase in volume and in the absence of other signs of recurrent disease, is advised.

\section{Lymph nodes $(\mathrm{N})$}

The incidence of loco-regional metastatic lymph nodes in HNRMS is low, about $10-20 \%$, tumour spread mainly being by way of vascular metastases in sarcomas $[4,13$, 44].

Enlarged lymph nodes may normally be observed in the retropharyngeal space and in the neck in children and

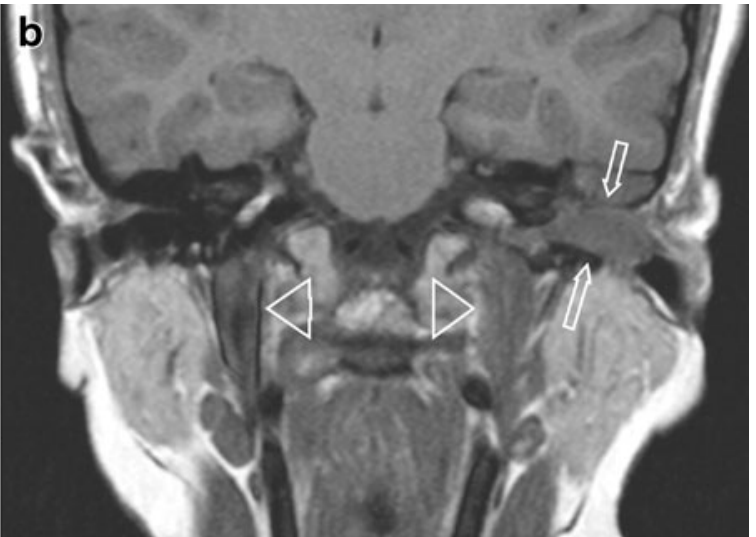

expansile mass within the EAC (arrows) extending into the tympanic cavity and slow flow within the left (and right) internal jugular vein (arrowheads). c Axial contrast-enhanced SE T1-W image shows that the mass strongly enhances and has invaded the middle ear cavity (large arrow). Without fat saturation the exact tumour extension is difficult to assess at the petrosal apex. In this case CT was helpful to exclude apical invasion (a) and confirm asymmetrical pneumatisation of the apices (small arrows)

therefore size criteria are less reliable than in adults. Unfortunately, no evidence-based papers regarding imaging appearance of malignant neck nodes in children exist and the findings in adult patients regard mostly patients with squamous cell carcinoma and cannot be used for children with sarcomas. There are, however, some important, empirical imaging findings in children with HNRMS. A hypoechoic, rounded neck node with obliteration of the fat centre is suspicious of a metastatic node and warrants fineneedle aspiration biopsy. Central necrosis is commonly seen in infectious disease (tuberculosis, cat-scratch disease) but rarely in malignant tumours.

For the assessment of lymph node metastases US, if needed with additional US-guided fine-needle cytology, remains our standard technique.

\section{Distant metastases (M)}

About $15 \%$ of children with HNRMS will have distant metastases at diagnosis [45]. Lung metastases are best identified with multidetector CT (MDCT), although it may 


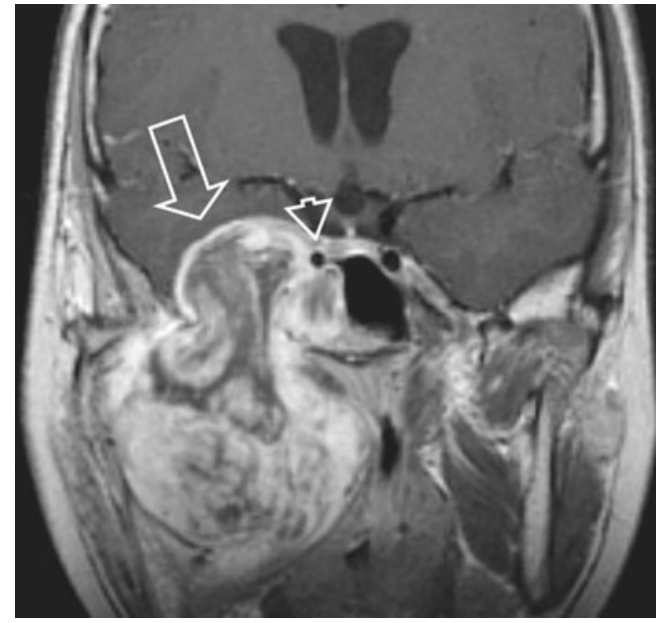

Fig. 5 Fourteen-year-old boy with a large MS tumour (eRMS). Coronal contrast-enhanced SE T1-W image shows extension through the massively enlarged foramen ovale into the cavernous sinus on the right side and asymmetry of the internal carotid artery by tumour compression (arrowhead). Note heterogeneous enhancement with strands of non-enhancing tissue within the tumour mass. There is also compression of the temporal lobe (arrow). No pathological enhancement of the adjacent brain, therefore no suspicion of direct tumour invasion of the brain

be impossible to differentiate benign from malignant nodules [8]. Bone metastases and metastases in other parts of the body are now best assessed by PET-CT or WBMRI because these techniques include the whole body, bone as well as soft tissues and parenchymal organs [14, 37, 38]. Equivocal lesions may be further evaluated by US or CT.

Brain metastases from HNRMS are rare and are mostly seen in patients with recurrent disease and distant metastatic disease in other parts of the body [46]. Imaging of the brain in search for brain metastases therefore is recommended only in clinically suspected patients [47].

\section{Imaging characteristics in HNRMS}

Most HNRMS are solitary, unilateral bulky lesions, although multifocal primary HNRMS has been reported [48].

Spin-echo T1-W sequences offer excellent contrast between the tumour and surrounding (obliterated) fat planes as well as the skull base in axial and coronal planes. SE T2$\mathrm{W}$ sequences may show a large variety of signal intensities (SI) of RMS in different patients and extremely low or high or intermediate SI, compared to muscle may be seen [13, 48-52]. Some tumours may appear relatively homogeneous and others are frankly heterogeneous, probably correlating with cellularity, tumour matrix and necrosis [22, 24, 25]. Understanding these MRI findings by correlating imaging with histopathological findings is subject of study in our group and will be reported elsewhere.
Although HNRMS may show a variety of enhancement patterns after IV contrast administration, the majority show moderate, homogeneous enhancement. Non-enhancing areas may be recognized after IV contrast administration, representing central necrosis or cavitation [49]. There seems to be no pathognomonic MRI appearance of HNRMS.

\section{Tumour locations and specific imaging concerns}

\section{PM HNRMS}

Parameningeal locations are in direct or near contact with the skull base. Tumours may originate from the nasopharynx (NP), the parapharyngeal space (PPS), the MS (formerly called the "infratemporal fossa"), the nasal cavity (Fig. 3), paranasal sinuses, the mastoid or the middle ear (Table 1) (Fig. 4). It may be difficult to assess the origin of a large lesion, but defining the centre of the bulk and analysing the way in which normal structures are displaced may help in defining the correct compartment [53]. PMRMS may show earlier spread to the skull base and intracranial compartment compared to other locations and accurate assessment of the local extent is one of the principal goals of imaging. Survival is worse and treatment options differ when there is skull base involvement or intracranial tumour extension $[4,54]$.

Skull base involvement may be well identified on noncontrast-enhanced SE T1-W sequences. Invasion of the sphenoid bone and the clivus and also the pterygoid bone and pterygoid plates may occur (Fig. 1). When a tumour is located in the masticator space the mandible and temporomandibular joint (TMJ) may be at risk. Tumour extension

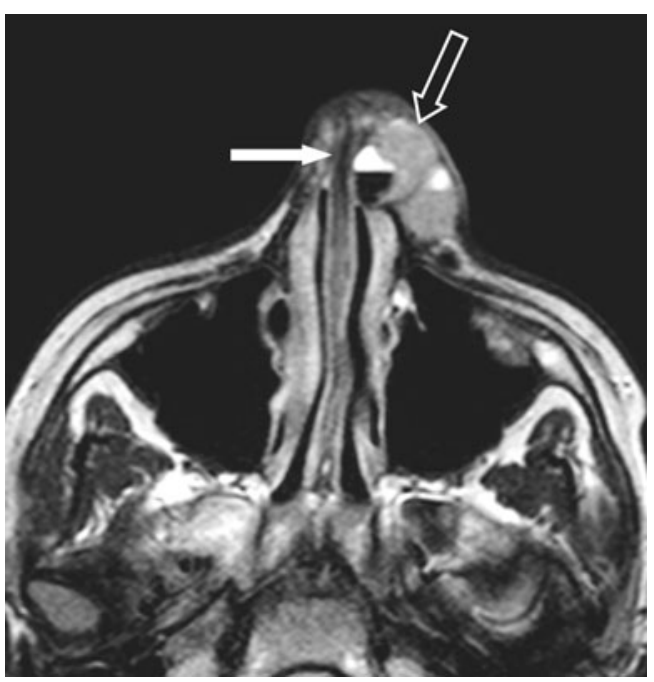

Fig. 6 Non-PM eRMS in a 7-year-old boy at the left side of the nose (open arrow). Axial SE T2-W image shows the small, superficial lesion with no deep extension. Fluid level (white arrow) represents haemorrhage after biopsy 

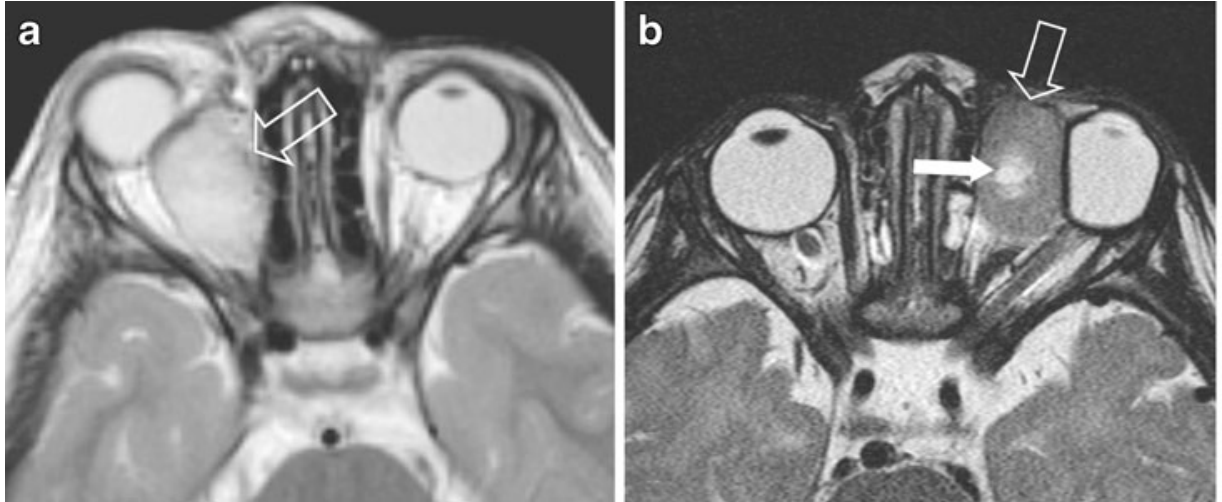

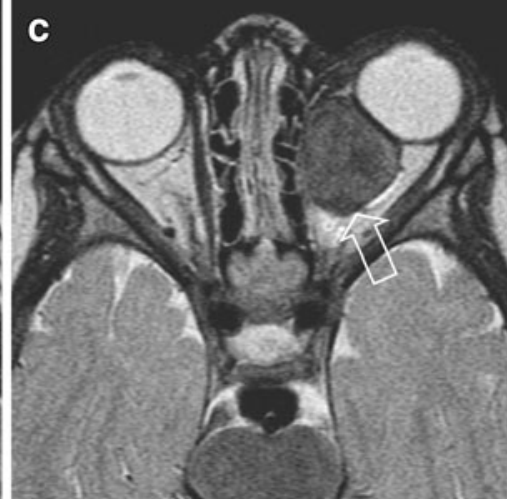

Fig. 7 Three examples of SE T2 appearances of different orbital tumours in young children, presenting with rapidly progressive proptosis. a 4-year old boy with right sided proptosis. SE T2 image shows a high signal lesion in the medial quadrant of the orbit (arrow), presumably extraconal. Biopsy: eRMS. b 1 -year old boy with rapidly progressive, left sided proptosis. SET2 shows intermediate signal intensity lesion (arrow), with central necrosis (white arrow), in the

Table 4 Differential diagnostic considerations

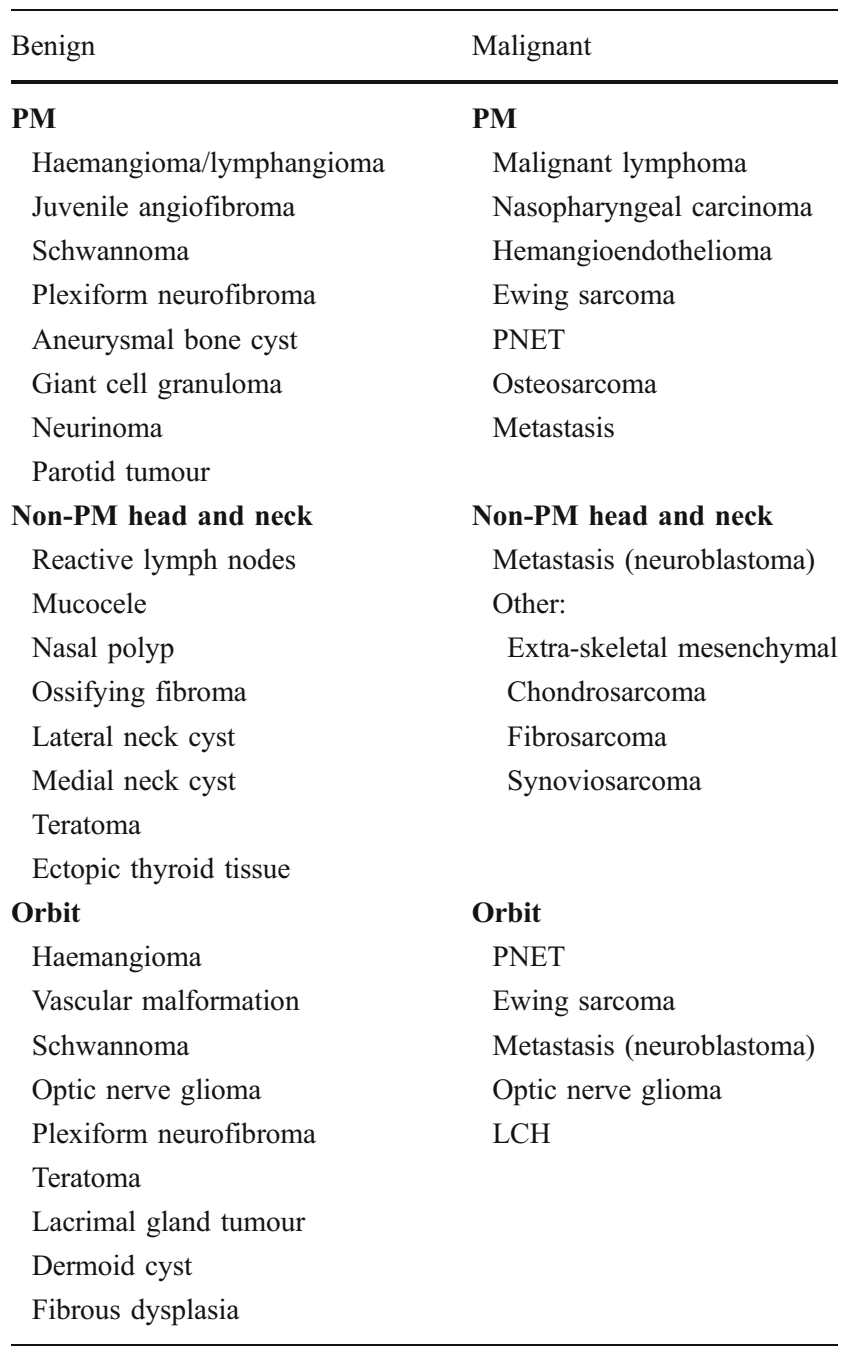

medial quadrant, compressing the lateral rectus muscle, presumably extraconal. Final diagnosis: alveolar RMS, rare at such a young age. c 2-year old girl with rapidly increasing left sided proptosis. SE T2 shows a very low signal intensity tumour in the medio-inferior part of the left orbit (arrow). Histology: primitive neuroendocrine tumour (PNET)

may be subtle and therefore one should look carefully for disappearance of normal fat planes and for abnormal contrast enhancement, comparing with the normal, non-involved side.

Contrast-enhanced images show pathological enhancement of the tumour, which may become more obvious when fat saturation is added. Subtle perineural extension becomes more obvious after IV contrast administration. Asymmetrical enlargement of the cavernous sinus with abnormal enhancement in the skull base foramina may be a subtle sign of intracranial tumour extension along the trigeminal nerve branches (see below).

Encasement of the internal carotid artery (Fig. 5) may be seen as well as compression of the jugular vein. Perivascular enhancement along the internal carotid artery near the skull base suggests tumour extension beyond the extracranial compartments and careful reappraisal of findings must be considered.

\section{Non-PM HNRMS}

These tumours are located in all other compartments except the orbit (Table 1). Careful assessment of local extension of oropharyngeal or oral cavity RMS into adjacent compartments, e.g., the PPS or MS, is mandatory, because this may change a low-risk location to a high-risk PM location. Intranasal locations may be purely non-PM (Fig. 6) but may become PM when the tumour abuts the skull base (Fig. 3).

Bone invasion of the hard palate may cause perineural spread along the palatine nerves and from there retrograde tumour extension may occur along the trigeminal branches and the vidian nerve into the cranial compartment, thereby altering the prognosis into a less favourable PM location.

Parotid HNRMS per se falls under the non-PM group, but in case of involvement of the facial nerve or extension 


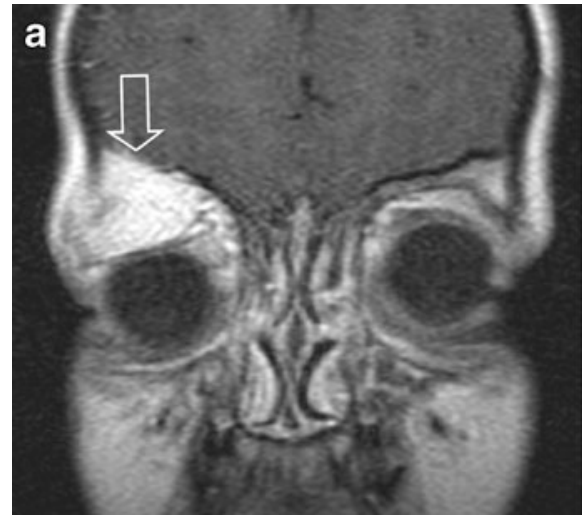

Fig. 8 Orbital eRMS with involvement of the roof of the orbit, a rare form of orbital RMS, in an 8-year old boy, presenting with orbital pain and proptosis. The bone invasion classifies the tumour as parameningeal, with a worse prognosis compared to orbital RMS. a Coronal post-contrast SET1 image shows a large, strongly enhancing lesion within the roof of the right orbit, extending into the superior extraconal compartment (open arrow), suggesting a primary bone tumour. Displacement of the eye ball downward and

into the PPS the tumour is classified as PM, with a worse prognosis.

RMS of the lower neck may extend into the mediastinum or, depending on the location, intraspinally. The latter finding will change prognosis because this is categorized as a PM location.

\section{Orbital RMS}

Periorbital RMS originates from the eyelids and may remain outside the orbit, i.e. in a preseptal location.

Intraorbital tumour includes extra- or intraconal tumour location. Most RMS is located within the superonasal quadrant of the orbit $[48,49]$. Assessment of its relationship with the extraocular muscles, the lacrimal gland, the lacrimal duct system, the optic nerve, the globe and the apex of the orbit is necessary (Table 1). Unlike PM locations, orbital RMS seldom invades the skull base or extends intracranially [11, 49]. Extension through the inferior or superior orbital fissure is rare, as is invasion of the adjacent paranasal sinuses.

Subtle bone deformity may be observed incidentally but frank bone destruction with involvement of the bone marrow and adjacent intracranial structures is rarely seen in orbital RMS (Fig. 7). Bone invasion will change the classification into a PM location. When bone destruction is suspected on MRI additional CT is recommended to confirm MRI findings and to exclude concomitant benign disease, e.g., fibrous dysplasia.

\section{Imaging differential diagnoses}

Tumours of the head and neck in the paediatric population are rare, representing about $2-3 \%$ of all head and neck tumours.

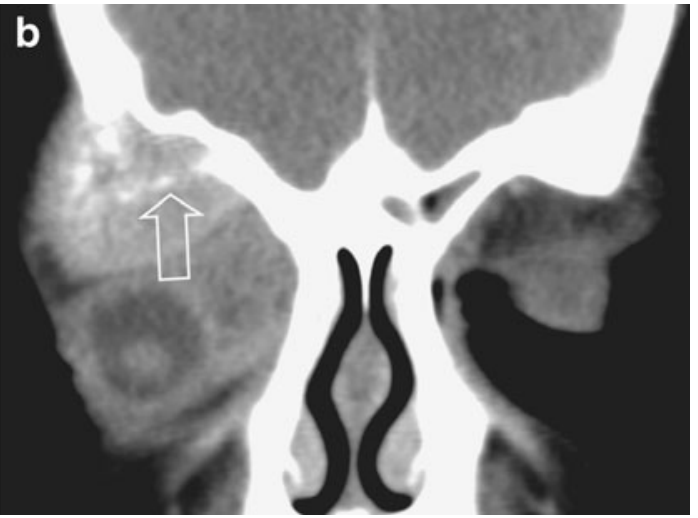

laterally. b Additional CT (coronal multiplanar reconstruction after i.v. contrast) confirmed bone involvement and local destruction with intraorbital extention (arrow). From the imaging it was impossible to differentiate between a primary bone tumour (MRI) or a primary (atypical) orbital location with adjacent bone invasion (CT). The location is not characteristic for RMS, which most often is within the medial quadrant. At surgery the lesion was considered a primary bone location. Histology: e-RMS

In many cases, imaging provides only a differential diagnosis and imaging findings should be correlated with clinical findings and discussed with the treating physician in each individual case to decide whether a biopsy should be taken or whether the child should be referred to a tertiary centre. It is beyond the purpose of this paper to provide an exhaustive overview of differential diagnostic considerations, but Table 4 may serve as a guideline.

\section{Malignant tumours other than RMS}

Apart from HNRMS, other rapidly progressive malignant childhood tumours of the head and neck may be encountered in young infants as well as older children. A few of these tumours will be briefly reviewed and differential diagnostic imaging findings will be addressed.

\section{Nasopharyngeal carcinoma}

Paediatric nasopharyngeal carcinoma (NPC) is rare with $<20 \%$ of NPC occurring in children [55]. Asymptomatic, large, unilateral or bilateral neck nodes are often the first clinical symptom. Bilateral large neck nodes are very uncommon in HNRMS, and this finding alone may direct the diagnostic work-up towards NPC (Fig. 8) [56].

\section{Malignant lymphoma}

Malignant lymphoma of the head and neck is the most common malignant head and neck tumour in children. It may involve Waldeyer ring, the MS, the paranasal sinuses and the orbits and often presents with bilaterally enlarged neck nodes $[57,58]$. Generally, malignant lymphoma has a 

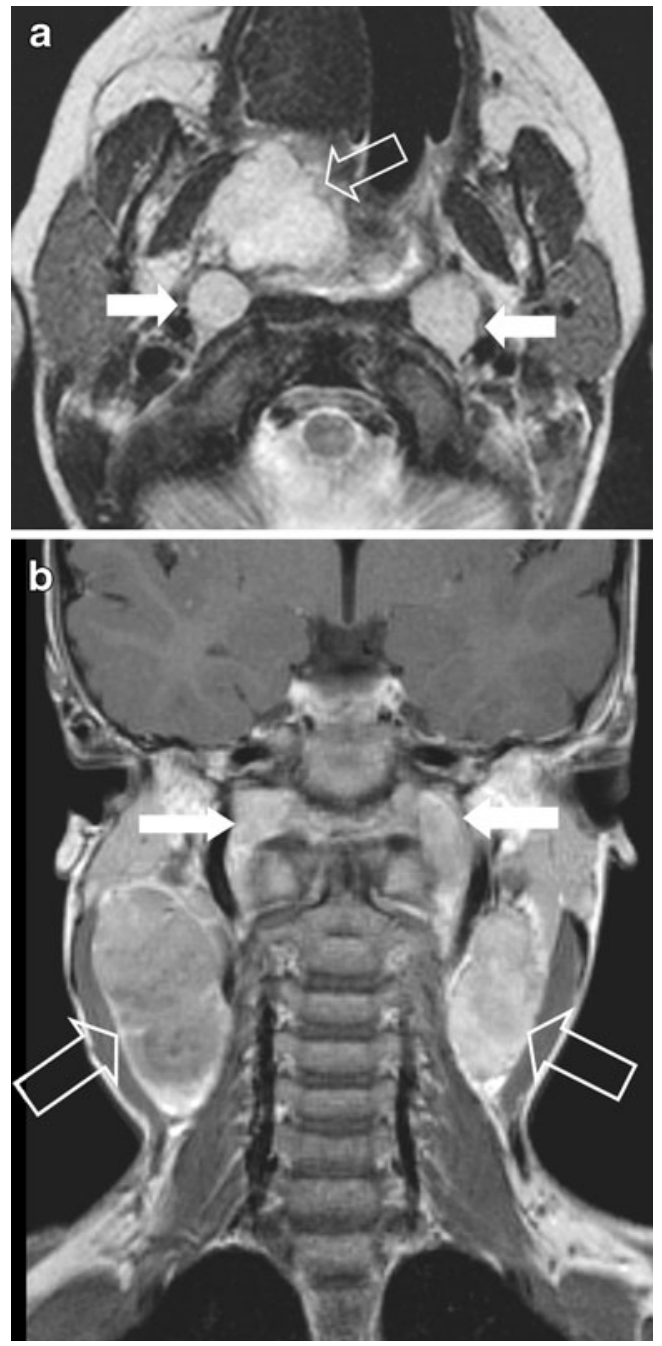

Fig. 9 Nasopharyngeal e-RMS in a 4-year old girl, presenting with massive cervical lymphadenopathy, suggesting nasopharyngeal cancer. a Axial SET2 weighted image showing a high signal lesion (arrow) within the right naso - oropharynx and enlarged retropharyngeal lymph nodes (white arrow). b Coronal SET2 image showing massive, bilateral cervical lymphadenopathy (open arrows \& white arrows). Final histology: e-RMS

surprisingly smooth appearance on imaging and shows moderate enhancement after IV contrast administration. Homogeneous enlargement of the lymphoid ring of Waldeyer in combination with unilateral or bilateral enlarged, homogeneous neck nodes may be seen in malignant lymphoma. Central necrosis in the neck nodes is seen in a small minority of patients.

Malignant lymphoma may also present with multiple bone lesions and in these cases Langerhans cell histiocytosis $(\mathrm{LCH})$ or metastases of neuroblastoma should also be considered.

A biopsy is required for final diagnosis, treatment being different for NPC, non-Hodgkin lymphoma (NHL), LCH and metastatic disease.
Ewing sarcoma/peripheral primitive neuroectodermal tumour

Ewing sarcoma of bone presents as a very aggressive mass lesion with permeative destruction of bone and invasion into surrounding tissues. Only 4\% of Ewing sarcoma of bone presents in the head and neck. Imaging may show a variety of signal intensities resembling NHL or even benign disease [59].

Peripheral primitive neuro-ectodermal tumour (pPNET) occurs at an even lower incidence and may present as a wellcircumscribed soft-tissue mass, with low-to-intermediate signal intensity on $\mathrm{T} 2-\mathrm{W}$ imaging and moderate enhancement after IV contrast administration [60]. A biopsy is mandatory to confirm the diagnosis as there are no characteristic imaging findings to differentiate these highly malignant tumours from other sarcomas, HNRMS, extranodal malignant lymphoma or even benign lesions.

\section{Salivary gland tumors}

Salivary gland tumours in children are rare, accounting for $<5 \%$ of all salivary gland tumours and $<8-10 \%$ of all paediatric head and neck tumours [61]. During infancy and early childhood the most common lesions are vascular malformations and lipomatous lesions. Sialoblastoma or embryoma is a histologically benign salivary gland tumour that is unique to children and has been reported only in newborns and neonates. About 25\% show features of malignancy but distant metastases are rare. There is a high risk of recurrence after incomplete surgical resection [61].

The majority of epithelial tumours occur after the age of 10-12 years and $>90 \%$ originate within the parotid gland. Unlike in adults, about $50 \%$ of parotid tumours in this group of children are malignant. Pleomorphic adenoma is the most common benign, while mucoepidermoid carcinoma is the most common malignant epithelial tumour [61]. Sarcomas are rare. A malignant salivary tumour in a younger child may behave as a highly aggressive tumour with a poor prognosis.

\section{Orbital tumours}

Rapidly progressive proptosis in a child is an indication for imaging. Although RMS is one of the most common orbital malignant tumours, other rapidly growing malignant lesions, such as malignant lymphoma, PNET and rhabdoid tumour should also be considered (Fig. 9).

Chloroma or granulocytic sarcoma is the commonest form of leukaemic involvement of the orbit, presenting as a solid mass in young children with acute myeloid leukaemia, which may precede the blast phase of the disease [62].

Primary bone lesions of the orbit may also result in proptosis. Fibrous dysplasia is a benign, expansile bone lesion involving the craniofacial bones and skull base in the young 

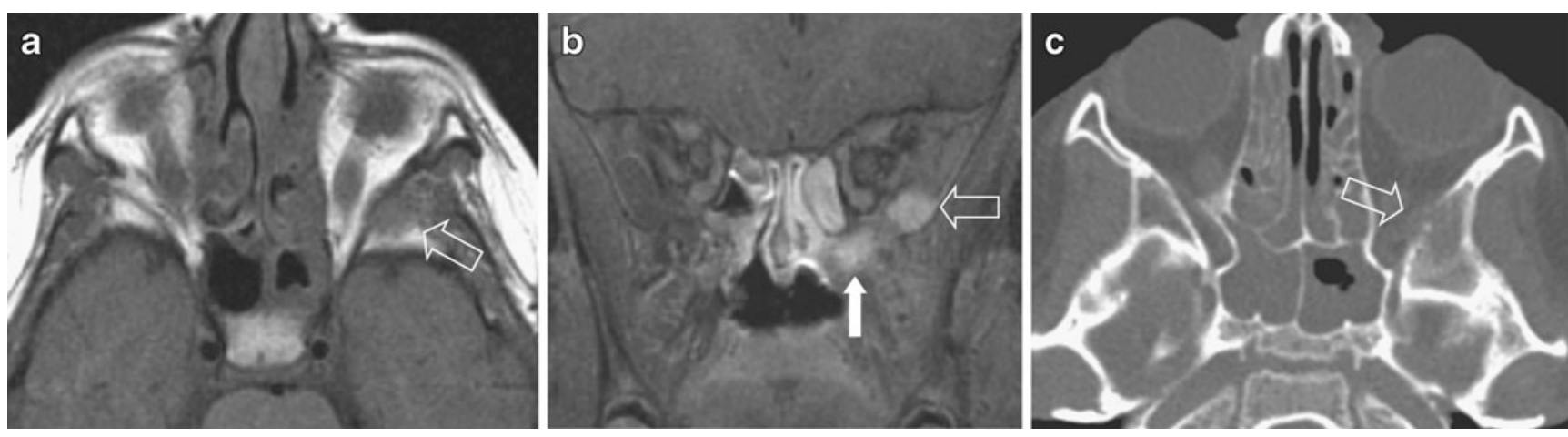

Fig. 10 Follow-up of an 8-year-old girl who had a parameningeal left sided eRMS 2 years previously with extension into the pterygopalatine fossa. After a full course of chemotherapy she underwent AMORE procedure. Clinically she is in complete remission 2 years after surgery. Postoperatively a slowly regressing, enhancing lesion has been present at the level of the left pterygoid, considered to be scar tissue. a Routine follow-up MRI at 2 years. Axial SE T1-W image

adolescent and adult patient. Additional CT may help establish the definitive diagnosis. Malignant bone tumours involving the orbit include Ewing sarcoma, malignant lymphoma or metastatic disease [63]. LCH is an uncommon disease characterized by clonal proliferation of Langerhans cells. Aetiology and pathology are still conjectural. LCH may present as a solitary bone lesion of the skull but also as multifocal or systemic disease and must be included in the differential diagnosis. Because of the space-occupying, systemic and invasive-destructive characteristics of the lesion(s), patients are often referred to the paediatric oncologist or radiotherapist for management advice. In all patients a biopsy is required to assess the final diagnosis.

\section{Treatment and prognosis}

Treatment of HNRMS requires a multidisciplinary approach, where chemotherapy, surgery and radiation therapy each has its own specific role. After the introduction of chemotherapy and improvement in local therapy and diagnostic techniques, survival has considerably improved over the last 30 years. Whereas in the past only $25 \%$ survived, nowadays patients with orbital primaries have up to $89 \%$ 3-year failure-free survival [3] and those with parameningeal locations show a 5 -year failure-free survival in $67 \%$ [4].

Orbital tumours have an excellent survival. Because the majority of patients have received chemotherapy and radiotherapy, an important number of patients encounter major late effects [64]. A joint European-North American analysis of their experience in orbital patients treated according to consecutive protocols (1979-1992) suggests that a subset of patients with orbital RMS can be cured without systematic local therapy, although the total burden of treatment must be taken into account when assessing the shows abnormal low signal within fat marrow of left zygoma with subtle intraorbital soft-tissue extension (arrow). b Coronal contrastenhanced fat-saturated SE T1-W image shows enhancement of the zygomatic lesion (open arrow). Inferomedially the residual disease (white arrow) is unchanged compared to previous MRI. c Additional axial CT 2 months later, shows local cortical destruction (arrow). Biopsy showed recurrent eRMS

implications for late sequelae [11]. Other approaches to minimize late effects in this patients group include the use of brachytherapy or proton therapy $[65,66]$.

Non-PM RMS also has a favourable outcome. More than $80 \%$ of patients with localized tumours are cured using surgery and multiagent chemotherapy, with or without radiotherapy [67]. Radiotherapy can be added for those with IRS-II or IRS-III lesions, depending on the achievement of complete remission by induction chemotherapy and cooperative group guidelines. More recent COG studies will answer the question whether early exposure to reduced dosages of alkylating agents may improve event-free survival.

Parameningeal-located tumours have the least favourable outcome of all HNRMS with a 5-year failure-free survival of $67 \%$ [4]. Prognosis is strongly related to PM subsite and presence or absence of meningeal involvement. Reviewing data from three consecutive COG studies, Raney et al. [4] identified three prognostic subsets: $20 \%$ of patients had tumours in the middle ear/mastoid, nasopharynx/nasal cavity (MEM, NNC) and PPS without meningeal involvement ( $20 \%$ of patients) and showed excellent long-term survival (92\% at 5 years). Fifty-six-percent of all PMN patients had either MEM- or NNC- or PPS-located tumours and meningeal involvement or had tumours in the paranasal sinuses or the pterygopalatine/infratemporal fossa (PNS, PIF) without meningeal involvement. For this subgroup of patients 5-year survival was intermediate, i.e. approximately $75 \%$. Patients with PNS or PIF tumours and meningeal involvement ( $24 \%$ of patients) had only a $57 \%$ survival rate at 5 years. Treatment outcome for patients treated after 1977 was considerably better. One of the most important reasons for this improvement was probably the availability of improved diagnostic imaging studies (CT and MRI) and the greater precision for determining appropriate volumes for radiotherapy. Subsequent COG studies have shown that 
whole-brain radiotherapy and intrathecal chemotherapy are not an essential part of the therapeutic regimen in patients with PMN RMS and therefore are no longer routinely used, resulting in an important reduction of late adverse effects in this patient category.

Regional lymph node involvement is relatively rare with an incidence of about $17 \%$ at diagnosis [4]. Involved lymph node sites should receive radiotherapy. Apart from the primary site, involvement of lymph nodes and IRSG, histology and age also contribute to overall outcome. The embryonal subtype has a better prognosis than alveolar RMS and young age $(<10$ years) is a favourable prognostic factor.

Fifteen-percent of patients with RMS have metastatic disease at first presentation. A recent pooled analysis from the United States and European co-operative groups has shown that the lungs are the most commonly involved distant site $(47 \%)$, followed by bone marrow $(38 \%)$, bone (34\%) and distant nodes (26\%). Prognosis appeared to be strongly correlated with age, site, bone and/or bone marrow involvement and the number of involved sites [45]. As the majority of HNRMS patients survive, prevention of late adverse effects is of utmost importance. However, adequate local therapy often includes radiotherapy in this patient group with a mean age of 5-6 years.

Therefore the ablative surgery, moulage brachytherapy and surgical reconstruction (AMORE) procedure was introduced in our institute in 1991 [54, 68]. After careful staging of children with histologically proven HNRMS, chemotherapy courses are given according to SIOP (or currently EpSSG) protocols. Imaging is repeated during chemotherapy and, after completion of the first cycle, residual disease is evaluated. Children who do not have evidence of skull base or intracranial extension of their residual tumour mass after induction chemotherapy are eligible for this procedure. Our results are encouraging in that children who were enrolled in the AMORE procedure have comparable survival and fewer complications than those who had external beam radiation therapy [69].

\section{Post-treatment follow-up}

During chemotherapy, in addition to a slow or rapid reduction in tumour volume, the tumour appearances may change. On MRI a change from high to low signal intensity on $\mathrm{T} 2-\mathrm{W}$ imaging as well as alteration of the enhancement pattern may be observed. The exact meaning of these changes is not well-understood, but probably relates to tumour necrosis, decrease in tumour vascularity and reactive changes of the surrounding tissues.

On the contrary, radiation and/or chemotherapy may invoke significant changes in tumour viability with only minimal changes in morphology, not recognized on routine
MRI [70]. An early study suggested that patients with residual lesions after completing treatment were more at risk of tumour recurrence than patients in whom no residual lesions are seen on cross-sectional imaging [71]. A more recent study, however, showed that tumour response to induction chemotherapy had no effect on event-free survival [72]. Furthermore, the presence or absence of a residual lesion after completion of therapy did not appear to have any impact on prognosis in a recent analysis by COG [73]. It would therefore be helpful to have a more sensitive tool to discriminate nonviable tumour necrosis or scar tissue from viable residual tumour.

Routine MRI techniques fail to differentiate tumour activity in a residual lesion from fibrosis, but a recent publication suggests excellent discrimination between viable and nonviable tissue by DWI [28]. DCE MRI may be useful to differentiate complete from partial remission after treatment but there is considerable overlap in enhancement patterns between benign and malignant tumours $[12,74]$. Up to now MR-spectroscopy remains unsuitable because of the complex anatomy of the head and neck area.

${ }^{18}$ FDG PET-CT seems to be very promising in determining the nature of residual lesions but has the disadvantages that it is a long examination and that it exposes young patients to ionizing radiation [20,75]. However, this may be a relative contra-indication: in terms of risk-benefit PETCT may be more useful to these children with a malignancy than omitting the PET-CT study. Although PET-CT seems to provide additional information, it is still optional in the international studies on RMS.

After surgery, interpretation of mass lesions may be extremely difficult on MRI or CT due to distortion or resection of soft tissues and bony landmarks, the replacement of excised structures with viable muscle during reconstructive surgery and the presence of bone and/or fat flaps, which may resemble tumour tissue. Therefore a baseline postoperative study is needed to avoid pitfalls during follow-up, which we routinely perform 3 months postoperatively. New techniques, such as DWI or PET-CT may help to differentiate residual active tumour from scar tissue after completion of treatment with the aim of thereby avoiding early recurrence.

During follow-up, imaging is routinely repeated every 3 months during the first year following end of treatment, every 4 months the 2 nd and 3 rd years and once per year for 2 more years.

Recurrent tumour is often suspected clinically but in a minority of patients treatment failure is first demonstrated by imaging (Fig. 10). Imaging may show subtle or large mass lesions, which may be solitary or multiple and a biopsy should be performed, whenever feasible, to assess the nature of the suspicious mass. New imaging techniques may help to confirm the nature of the mass in patients in whom a biopsy is hazardous. 


\section{Conclusion}

MR imaging plays a crucial role in the primary evaluation of head and neck tumours in the paediatric population as it offers an exquisite depiction of soft-tissue tumours of the face and orbit, the deep compartments of the head and neck and the skull base. However, in the absence of characteristic imaging appearances, histological diagnosis should be obtained for all mass lesions whenever feasible (i.e. without serious complications, e.g., blindness, gross haemorrhage, cranial nerve damage). When no biopsy can be performed, close follow-up by imaging should be considered. Rapid progression can be differentiated from no perceivable growth and imaging may thereby guide aggressive diagnostic and treatment strategies.

Staging of HNRMS is best achieved by a combination of imaging techniques, where MRI offers a major contribution to assessing local extent of the primary tumour and MRI and US play an invaluable role in the assessment of cervical lymph nodes. According to recent publications WBMRI and PET-CT can make a major contribution to the assessment of distant metastases.

After completion of treatment, MRI depicts the presence or absence of residual masses and postsurgical anatomical changes and thus serves as an excellent baseline for longterm follow-up. Baseline MRI 3 months after extensive surgery and/or radiotherapy may help to identify residual/ recurrent disease at an earlier stage.

In the near future new MRI techniques such as DWI, WBMRI and/or PET-CT may play an important role in monitoring initial therapy, differentiating the responders from the nonresponders on chemotherapy, and may help improve noninvasive discrimination between residual viable and non-viable tumour masses in patients treated for craniofacial RMS.

Open Access This article is distributed under the terms of the Creative Commons Attribution Noncommercial License which permits any noncommercial use, distribution, and reproduction in any medium, provided the original author(s) and source are credited.

\section{References}

1. Miller RW, Young JL Jr, Novakovic B (1995) Childhood cancer. Cancer 75:395-405

2. Sengupta S, Pal R (2009) Clinicopathological correlates of pediatric head and neck cancer. J Cancer Res Ther 5:181-185

3. Crist WM, Anderson JR, Meza JL et al (2001) Intergroup rhabdomyosarcoma study-IV: results for patients with nonmetastatic disease. J Clin Oncol 19:3091-3102

4. Raney RB, Meza J, Anderson JR et al (2002) Treatment of children and adolescents with localized parameningeal sarcoma: experience of the Intergroup Rhabdomyosarcoma Study Group protocols IRS-II through -IV, 1978-1997. Med Pediatr Oncol 38:22-32

5. Lobe TE, Wiener ES, Hays DM et al (1994) Neonatal rhabdomyosarcoma: the IRS experience. J Pediatr Surg 29:1167-1170

6. Ragab AH, Heyn R, Tefft M et al (1986) Infants younger than 1 year of age with rhabdomyosarcoma. Cancer 58:2606-2610

7. Dickson PV, Davidoff AM (2006) Malignant neoplasms of the head and neck. Semin Pediatr Surg 15:92-98

8. Van Rijn RR, Wilde JC, Bras J et al (2008) Imaging findings in noncraniofacial childhood rhabdomyosarcoma. Pediatr Radiol 38:617-634

9. Stevens MC, Rey A, Bouvet N et al (2005) Treatment of nonmetastatic rhabdomyosarcoma in childhood and adolescence: third study of the International Society of Paediatric Oncology-SIOP Malignant Mesenchymal Tumor 89. J Clin Oncol 23:2618-2628

10. Hicks J, Flaitz C (2002) Rhabdomyosarcoma of the head and neck in children. Oral Oncol 38:450-459

11. Oberlin O, Rey A, Anderson J et al (2001) Treatment of orbital rhabdomyosarcoma: survival and late effects of treatment - results of an international workshop. J Clin Oncol 19:197-204

12. Razek AA, Elsorogy LG, Soliman NY et al (2009) Dynamic susceptibility contrast perfusion MR imaging in distinguishing malignant from benign head and neck tumors: A pilot study. Eur J Radiol Aug 18 [Epub ahead of print]

13. Kim EE, Valenzuela RF, Kumar AJ et al (2000) Imaging and clinical spectrum of rhabdomyosarcoma in children. Clin Imaging 24:257-262

14. Daldrup-Link HE, Franzius C, Link TM et al (2001) Whole-body MR imaging for detection of bone metastases in children and young adults: comparison with skeletal scintigraphy and FDG PET. AJR 177:229-236

15. Goo HW, Choi SH, Ghim T et al (2005) Whole-body MRI of paediatric malignant tumours: comparison with conventional oncological imaging methods. Pediatr Radiol 35:766-773

16. van den Brekel MW, Castelijns JA, Stel HV et al (1993) Modern imaging techniques and ultrasound-guided aspiration cytology for the assessment of neck node metastases: a prospective comparative study. Eur Arch Otorhinolaryngol 250:11-17

17. Volker T, Denecke T, Steffen I et al (2007) Positron emission tomography for staging of pediatric sarcoma patients: results of a prospective multicenter trial. J Clin Oncol 25:5435-5441

18. Abdel Razek AA, Soliman NY, Elkhamary S et al (2006) Role of diffusion-weighted MR imaging in cervical lymphadenopathy. Eur Radiol 16:1468-1477

19. Mody RJ, Bui C, Hutchinson RJ et al (2010) FDG PET imaging of childhood sarcomas. Pediatr Blood Cancer 54:222-227

20. Al-Ibraheem A, Buck A, Krause BJ et al (2009) Clinical Applications of FDG PET and PET/CT in Head and Neck Cancer. J Oncol Aug 20 [Epub ahead of print]

21. Abdel Razek AA, Kandeel AY, Soliman N et al (2007) Role of diffusion-weighted echo-planar MR imaging in differentiation of residual or recurrent head and neck tumors and posttreatment changes. AJNR 28:1146-1152

22. Humphries PD, Sebire NJ, Siegel MJ et al (2007) Tumors in pediatric patients at diffusion-weighted MR imaging: apparent diffusion coefficient and tumor cellularity. Radiology 245:848-854

23. Hermans R (2008) Posttreatment imaging in head and neck cancer. Eur J Radiol 66:501-511

24. Srinivasan A, Dvorak R, Perni K et al (2008) Differentiation of benign and malignant pathology in the head and neck using $3 \mathrm{~T}$ apparent diffusion coefficient values: early experience. AJNR 29:40-44

25. Sakamoto J, Yoshino N, Okochi K et al (2009) Tissue characterization of head and neck lesions using diffusion-weighted MR imaging with SPLICE. Eur J Radiol 69:260-268

26. King AD, Ahuja AT, Yeung DK et al (2007) Malignant cervical lymphadenopathy: diagnostic accuracy of diffusion-weighted MR imaging. Radiology 245:806-813 
27. Vandecaveye V, De Keyzer F, Vander Poorten V et al (2009) Head and neck squamous cell carcinoma: value of diffusion-weighted MR imaging for nodal staging. Radiology 251:134-146

28. Abdel Razek AAK, Megahed Alem S, Denewer A et al (2008) Role of diffusion-weighted magnetic resonance imaging in differentiation between the viable and necrotic parts of head and neck tumors. Acta Radiol 49:364-370

29. Kataoka M, Ueda H, Koyama T et al (2005) Contrast-enhanced volumetric interpolated breath-hold examination compared with spin-echo T1-weighted imaging of head and neck tumors. AJR 184:313-319

30. Lavini C, Pikaart BP, de Jonge MC et al (2009) Region of interest and pixel-by-pixel analysis of dynamic contrast enhanced magnetic resonance imaging parameters and time-intensity curve shapes: a comparison in chondroid tumors. Magn Reson Imaging 27:62-68

31. van Rijswijk CS, Geirnaerdt MJ, Hogendoorn PC et al (2003) Dynamic contrast-enhanced MR imaging in monitoring response to isolated limb perfusion in high-grade soft tissue sarcoma: initial results. Eur Radiol 13:1849-1858

32. Schuetze SM, Baker LH, Benjamin RS et al (2008) Selection of response criteria for clinical trials of sarcoma treatment. Oncologist 13:32-40

33. Voss SD, Reaman GH, Kaste SC et al (2009) The ALARA concept in pediatric oncology. Pediatr Radiol 39:1142-1146

34. Brenner D, Elliston C, Hall E et al (2001) Estimated risks of radiation induced fatal cancer from pediatric CT. AJR 176:289-296

35. Paterson A, Frush DP, Donnelly LF (2001) Helical CT of the body: are settings adjusted for pediatric patients? AJR 176:297-301

36. Jager PL, Hoekstra HJ, Leeuw J et al (2000) Routine bone scintigraphy in primary staging of soft tissue sarcoma; Is it worthwhile? Cancer 89:1726-1731

37. Tateishi U, Hosono A, Makimoto A et al (2009) Comparative study of FDG PET/CT and conventional imaging in the staging of rhabdomyosarcoma. Ann Nucl Med 23:155-161

38. Klem ML, Grewal RK, Wexler LH et al (2007) PET for staging in rhabdomyosarcoma: an evaluation of PET as an adjunct to current staging tools. J Pediatr Hematol Oncol 29:9-14

39. Jungehuelsing M, Sittel C, Fischbach R et al (2000) Limitations of magnetic resonance imaging in the evaluation of perineural tumor spread causing facial nerve paralysis. Arch Otolaryngol Head Neck Surg 126:506-510

40. Majoie CB, Hulsmans FJ, Verbeeten B Jr et al (1997) Perineural tumor extension along the trigeminal nerve: magnetic resonance imaging findings. Eur J Radiol 24:191-205

41. Nemzek WR, Hecht S, Gandour-Edwards R et al (1998) Perineural spread of head and neck tumors: how accurate is MR imaging? AJNR 19:701-706

42. Sigal R, Monnet O, de Baere T et al (1992) Adenoid cystic carcinoma of the head and neck: evaluation with MR imaging and clinicalpathologic correlation in 27 patients. Radiology 184:95-101

43. Borges A, Casselman J (2007) Imaging the cranial nerves: part II: primary and secondary neoplastic conditions and neurovascular conflicts. Eur Radiol 17:2332-2344

44. Leong SP, Cady B, Jablons DM et al (2006) Clinical patterns of metastasis. Cancer Metastasis Rev 25:221-232

45. Oberlin O, Rey A, Lyden E et al (2008) Prognostic factors in metastatic rhabdomyosarcomas: results of a pooled analysis from United States and European cooperative groups. J Clin Oncol 26:2384-2389

46. Paulino AC, Bauman N, Simon JH et al (2003) Local control of parameningeal rhabdomyosarcoma: outcomes in noncomplete responders to chemoradiation. Med Pediatr Oncol $41: 118-122$

47. Spunt SL, Anderson JR, Teot LA et al (2001) Routine brain imaging is unwarranted in asymptomatic patients with rhabdo- myosarcoma arising outside of the head and neck region that is metastatic at diagnosis: a report from the Intergroup Rhabdomyosarcoma Study Group. Cancer 92:121-125

48. Sohaib SA, Moseley I, Wright JE (1998) Orbital rhabdomyosarcoma - the radiological characteristics. Clin Radiol 53:357-362

49. Shields CL, Shields JA, Honavar SG et al (2001) Clinical spectrum of primary ophthalmic rhabdomyosarcoma. Ophthalmology 108:2284-2292

50. Vanel D, Shapeero LG, de Baere T et al (1994) MR imaging in the follow-up of malignant and aggressive soft-tissue tumors: results of 511 examinations. Radiology 190:263-268

51. Yang WT, Kwan WH, Li CK et al (1997) Imaging of pediatric head and neck rhabdomyosarcomas with emphasis on magnetic resonance imaging and a review of the literature. Pediatr Hematol Oncol 14:243-257

52. Yousem DM, Lexa FJ, Bilaniuk LT et al (1990) Rhabdomyosarcomas in the head and neck: MR imaging evaluation. Radiology 177:683-686

53. Harnsberger HR, Osborn AG (1991) Differential diagnosis of head and neck lesions based on their space of origin. 1. The suprahyoid part of the neck. AJR 157:147-154

54. Buwalda J, Schouwenburg PF, Blank LE et al (2003) A novel local treatment strategy for advanced stage head and neck rhabdomyosarcomas in children: results of the AMORE protocol. Eur J Cancer 39:1594-1602

55. Wenig BM, Heffness CS (2008) Non-epithelial malignant salivary gland tumours. In: Atlas of head and neck pathology, 2nd edn. Elsevier, Amsterdam, p 685

56. Mafee MF (2005) The nasopharynx - nasopharyngeal carcinoma. Valvassori's imaging of the head and neck, 2nd edn. Thieme, NY, USA, p 569

57. Demirci H, Shields CL, Karatza EC et al (2008) Orbital lymphoproliferative tumors: analysis of clinical features and systemic involvement in 160 cases. Ophthalmology 115:1626-1631

58. Toma P, Granata C, Rossi A et al (2007) Multimodality imaging of Hodgkin disease and non-Hodgkin lymphomas in children. Radiographics 27:1335-1354

59. Cotterill SJ, Ahrens S, Paulussen M et al (2000) Prognostic factors in Ewing's tumor of bone: analysis of 975 patients from the European Intergroup Cooperative Ewing's Sarcoma Study Group. J Clin Oncol 18:3108-3114

60. van den Berg H, Heinen RC, van der Pal HJ et al (2009) Extraosseous Ewing sarcoma. Pediatr Hematol Oncol 26:175-185

61. Bradley P, McClelland L, Mehta D (2007) Paediatric salivary gland epithelial neoplasms. ORL J Otorhinolaryngol Relat Spec 69:137-145

62. Noh BW, Park SW, Chun JE et al (2009) Granulocytic Sarcoma in the Head and Neck: CT and MR Imaging Findings. Clin Exp Otorhinolaryngol 2:66-71

63. Vosoghi H, Rodriguez-Galindo C, Wilson MW (2009) Orbital involvement in langerhans cell histiocytosis. Ophthalmic Plast Reconstr Surg 25:430-433

64. Raney RB, Anderson JR, Kollath J et al (2000) Late effects of therapy in 94 patients with localized rhabdomyosarcoma of the orbit: Report from the Intergroup Rhabdomyosarcoma Study (IRS)-III, 1984-1991. Med Pediatr Oncol 34:413-420

65. Blank LE, Koedooder K, van der Grient HN et al (2009) Brachytherapy as Part of the Multidisciplinary Treatment of Childhood Rhabdomyosarcomas of the Orbit. Int $\mathrm{J}$ Radiat Oncol Biol Phys Oct 26 [Epub ahead of print]

66. Yock T, Schneider R, Friedmann A et al (2005) Proton radiotherapy for orbital rhabdomyosarcoma: clinical outcome and a dosimetric comparison with photons. Int J Radiat Oncol Biol Phys 63:1161-1168

67. Pappo AS, Meza JL, Donaldson SS et al (2003) Treatment of localized nonorbital, nonparameningeal head and neck rhabdo- 
myosarcoma: lessons learned from intergroup rhabdomyosarcoma studies III and IV. J Clin Oncol 21:638-645

68. Schouwenburg PF, Kupperman D, Bakker FP et al (1998) New combined treatment of surgery, radiotherapy, and reconstruction in head and neck rhabdomyosarcoma in children: the AMORE protocol. Head Neck 20:283-292

69. Blank LE, Koedooder K, Pieters BR et al (2009) The AMORE protocol for advanced-stage and recurrent nonorbital rhabdomyosarcoma in the head-and-neck region of children: a radiation oncology view. Int J Radiat Oncol Biol Phys 74:1555-1562

70. McCarville MB, Christie R, Daw NC et al (2005) PET/CT in the evaluation of childhood sarcomas. AJR 184:1293-1304

71. Gilles R, Couanet D, Chevret S et al (1991) Importance of a posttherapeutic residue in the prognosis of head and neck rhabdomyosarcoma in children. Eur J Radiol 13:187-191

72. Burke M, Anderson JR, Kao SC et al (2007) Assessment of response to induction therapy and its influence on 5-year failure- free survival in group III rhabdomyosarcoma: the Intergroup Rhabdomyosarcoma Study-IV experience - a report from the Soft Tissue Sarcoma Committee of the Children's Oncology Group. J Clin Oncol 25:4909-4913

73. Rodeberg DA, Stoner JA, Hayes-Jordan A et al (2009) Prognostic significance of tumor response at the end of therapy in group III rhabdomyosarcoma: a report from the children's oncology group. J Clin Oncol 27:3705-3711

74. De Schepper AM, Bloem JL (2007) Soft tissue tumors: grading, staging, and tissue-specific diagnosis. Top Magn Reson Imaging 18:431-444

75. Peng F, Rabkin G, Muzik O (2006) Use of 2-deoxy-2-[F-18]fluoro-D-glucose positron emission tomography to monitor therapeutic response by rhabdomyosarcoma in children: report of a retrospective case study. Clin Nucl Med 31:394-397

76. Bisogno G, Bergeron C, Jenney M et al (2005) EpSSG RMS 2005; a protocol for non-metastatic rhabdomyosarcoma. 2005 Jun 1, Padova 